\title{
Optical Characterization of an X-ray Zoom Lens.
}

$\underline{\text { Elisa Kornemann }}^{1, *}$, Ottó Márkus ${ }^{1}$, Alexander Opolka ${ }^{1}$, Kawal Sawhney ${ }^{2}$, Angelica Cecilia ${ }^{3}$, Mathias Hurst $^{3}$, Tilo Baumbach ${ }^{3}$, Arndt Last ${ }^{1}$ and Jürgen Mohr ${ }^{1}$

1. IMT, Karlsruhe Institute of Technology (KIT), Eggenstein-Leopoldshafen, Germany

2. Diamond Light Source, Harwell Science and Innovation Campus, Didcot, United Kingdom

3. IPS, Karlsruhe Institute of Technology (KIT), Eggenstein-Leopoldshafen, Germany

* elisa.kornemann@kit.edu

We present results on the characterization of an X-ray zoom lens [1] via full field microscopy and enlarged cone beam projection. The X-ray zoom lens is fabricated from Compound Refractive X-ray Lenses (CRLs) made out of SU-8 negative photoresist by deep X-ray lithography [2]. The commonly known CRLs with variable focal length like the transfocator [3] or the F-switch [4] are mainly used for beam conditioning with focal length in the meter range and focal spot size in the tens- to hundredmicrometer range. Our X-ray zoom lens is capable of changing the focal length from centimeter up to meter range very fast. It shows a focal spot size in the sub-micrometer range with at the same time large field of view (FoV) and therefore can be used as an objective lens. It can be installed easily in different experimental setups due to its compactness of about one liter.

The proof of concept and characterization regarding focal lengths and focal spot sizes of such an X-ray zoom lens at ID01, ESRF, and P05, PETRA III, was reported recently [1]. For the first time this lens was built up in a full field microscope setup at the IMAGE beamline at the KIT synchrotron. The setup contains a Siemens-star X500-200-30 as test structure, the X-ray zoom lens as objective and a PCO4000 detector. At a photon energy of $E_{\mathrm{Ph}}=17.5 \mathrm{keV}$ the X-ray zoom lens showed a focal length of $f=324 \mathrm{~mm}$ and a magnification factor of $M=6$. Our aim was to image a sample fast with different energies without touching the microscope setup. Therefore, we adapted the number of the lens elements in the beam to keep the focal distance constant. Thus, the magnification factor $M$ in the microscope setup was constant without repositioning any optical element. In Figure 1 two different X-ray lens configurations are compared; one with $N=36$ lens elements in the beam at $E_{\mathrm{Ph}}=17.5 \mathrm{keV}$ and the other with $N=28$ lens elements in the beam at $E_{\mathrm{Ph}}=15.43 \mathrm{keV}$. With a large FoV of $185 \mu \mathrm{m}$ x $200 \mu \mathrm{m}$ it was possible to resolve the complete Siemens-star (Figure 1 (a), (b)) with smallest feature size of $0.5 \mu \mathrm{m}$ (Figure 1 (c),(d)) and show an equivalent quality in resolution, sharpness and magnification. This characteristic of our X-ray zoom lens opens the possibility of fast spectroscopy measurements in a wide $\mathrm{X}$-ray regime.

Additionally, we demonstrated for the first time the possibility to investigate a sample with different magnification $M$ and FoV without touching the experiment. We used our X-ray zoom lens with eccentrics as actuators in an enlarged cone beam projection setup at B16, beamline at DIAMOND. This lens showed a focal spot size of $\sigma=0.6 \mu \mathrm{m}$ (FWHM) measured with a $200 \mu \mathrm{m}$ thick gold wire in a knife edge scan. The imaged sample was a grating with $2.3 \mu \mathrm{m}$ period and with $11 \mu \mathrm{m}$ Nickel on a $525 \mu \mathrm{m} \mathrm{Si}$ substrate (fabricated at KIT/IMT). It was positioned $s_{\mathrm{d}}=534 \mathrm{~mm}$ behind the X-ray zoom lens, followed by a PCO4000 detector $646 \mathrm{~mm}$ behind the sample. The photon energy stays constant at $E_{\mathrm{Ph}}=19 \mathrm{keV}$ as well as the complete enlarged cone beam projection setup. Only the number of lens elements is changed to five different configurations, which change the focal length $f$ and therefore the magnification factor $M$ as well as the FoV as shown in Figure 2. In this case the focal length was changed from the minimum of 
$f_{1}=190 \mathrm{~mm}$ (Figure 2a) to $f_{5}=308 \mathrm{~mm}$ (Figure 2e) in five steps in a short time keeping the position of interest. This results in a change of the magnification by a factor 1.5. With this lens layout one can even go to a focal length up to $f=7.7 \mathrm{~m}$. This X-ray zoom lens allows to analyze the sample in a general overview to identify deviations from the expected image, which than can be investigated in more detail by zooming in without changing the measurement setup.

[1] E. Kornemann et al, Optics Express 25 (2017), pp. 22455-22466.

[2] V. Nazmov et al, Microsystem Technologies 10 (2004), pp. 716-721.

[3] G. B. M. Vaughan et al, J. Synchrotron Rad. 18 (2011), pp. 125-133.

[4] G. M. A. Duller et al, Conf. Proc. MEDSI'16 (2017), paper WEPE22, pp. 345-347.

[5] The authors thank the Karlsruhe Nano Micro Facility (KNMF) for the possibility to fabricate polymer X-ray lenses. We acknowledge the support of the Karlsruhe School of Optics and Photonics (KSOP). The research has been supported by the project CALIPSOplus under the Grant Agreement 730872 from the EU Framework Programme for Research and Innovation HORIZON 2020.
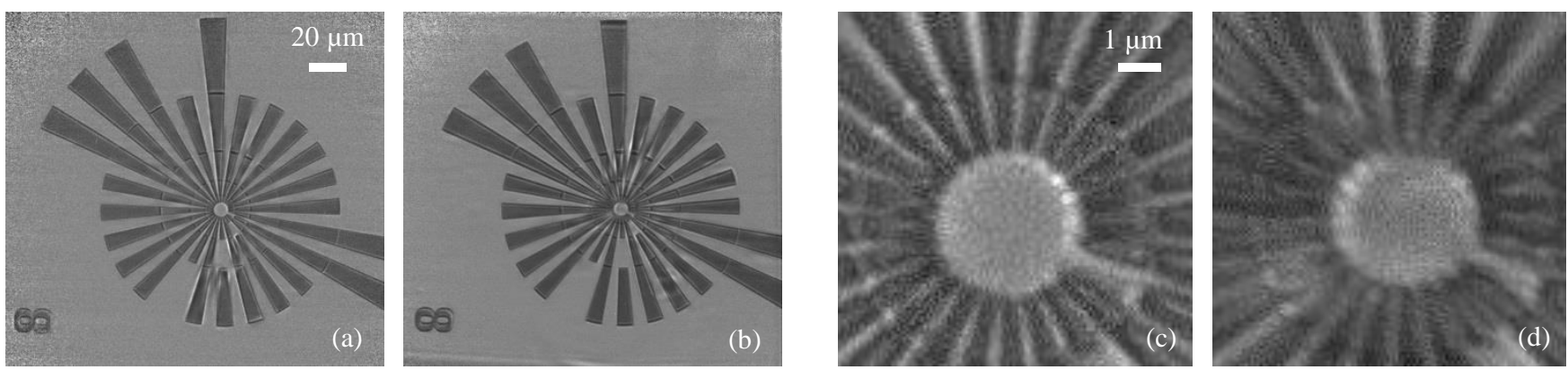

Figure 1. Full field microscopy imaging of a Siemens-star X500-200-30 with FoV $=185 \mu \mathrm{m}$ x $200 \mu \mathrm{m}$ in (a) and (b) at IMAGE, KIT Synchrotron. In the detail view of (c) and (d) the smallest features with $0.5 \mu \mathrm{m}$ are shown. The sample is investigated with two different energies and the zoom lens adapts the configurations to achieve sharp images in equal magnification:

In (a) and (c) $N=36, E_{\mathrm{Ph}}=17.5 \mathrm{keV}$, in (b) and (d) $N=28, E_{\mathrm{Ph}}=15.43 \mathrm{keV}$.
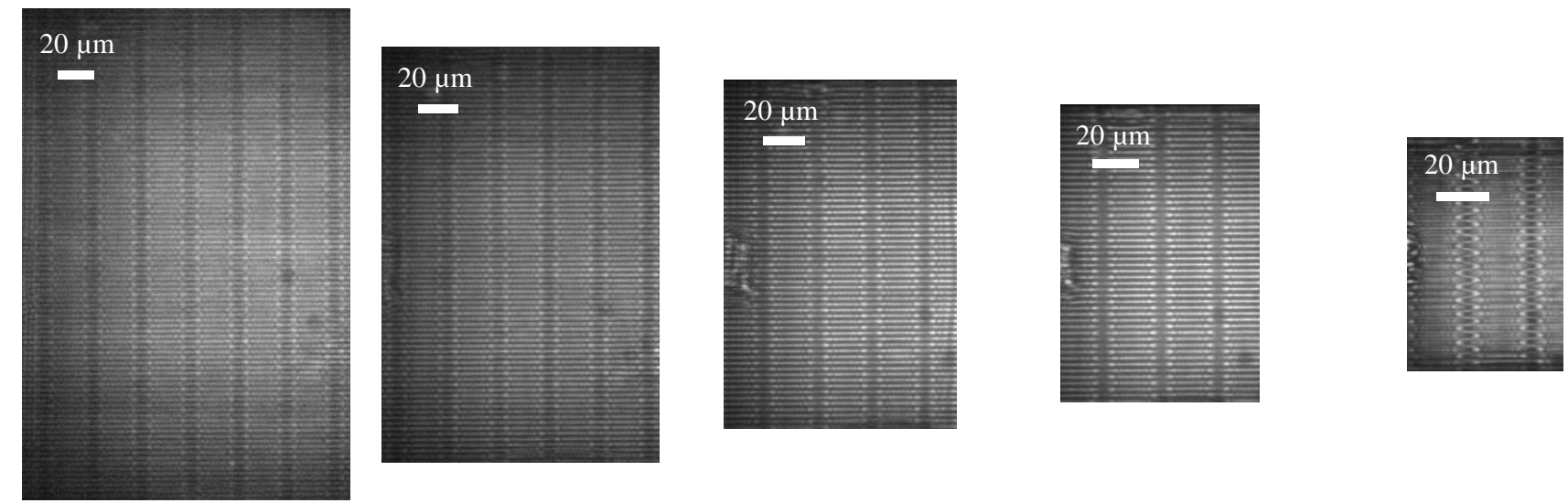

a) $f_{1}=190 \mathrm{~mm}$

b) $f_{2}=211 \mathrm{~mm}$

$N=44$ (config. \#1)

$N=38$ (config. \#2)

$M_{1}=1.88$

$M_{2}=2.00$

c) $f_{3}=242 \mathrm{~mm}$

$N=34$ (config. \#3)

$M_{3}=2.21$

d) $f_{4}=270 \mathrm{~mm}$

e) $f_{5}=308 \mathrm{~mm}$

$N=32$ (config. \#4)

$N=28$ (config. \#5)

$M_{4}=2.45$

$M_{5}=2.86$

$\mathrm{FoV}=(219 \times 328) \mu \mathrm{m}^{2}$

$\mathrm{FoV}=(174 \times 261) \mu \mathrm{m}^{2}$

$\mathrm{FoV}=(133 \times 199) \mu \mathrm{m}^{2}$

$\mathrm{FoV}=(102 \times 153) \mu \mathrm{m}^{2}$

$\mathrm{FoV}=(68 \mathrm{x} 103) \mu \mathrm{m}^{2}$

Figure 2. Enlarged cone beam projection at $E_{\mathrm{Ph}}=19 \mathrm{keV}$ at B16, DIAMOND. A $2.3 \mu \mathrm{m}$ period grating (11 $\mu \mathrm{m}$ Nickel on a $525 \mu \mathrm{m} \mathrm{Si-substrate)} \mathrm{is} \mathrm{investigated} \mathrm{with} \mathrm{the} \mathrm{X-ray} \mathrm{zoom} \mathrm{lens} \mathrm{in} \mathrm{five} \mathrm{configurations}$ a)-e) resulting in five images of different magnification factor $M$ and field of view. 